\title{
«Die 75 feiern und dann sterben» - wirklich?
}

\author{
Jean Martin \\ Dr. med., Mitglied der Redaktion, ehemaliges Mitglied der Nationalen Ethikkommission
}

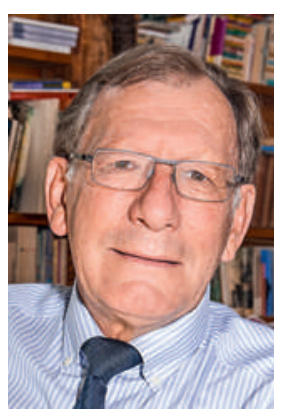

Ezekiel J. Emanuel, 58 Jahre, ist ein bekannter, im Bereich der Bioethik tätiger Onkologe in den USA. Er stammt aus einer aussergewöhnlichen Familie (sein Bruder Rahm war Berater von Präsident Obama und ist Bürgermeister von Chicago). Anfang Juni war er in Genf und sprach zu einem Thema, zu dem er bereits im bekannten Ostküstenmagazin The Atlantic referiert hatte [1].

«Unsere Lebenserwartung hat sich deutlich nach hinten verschoben. Dies ist sicherlich eine positive Errungenschaft. Gleichzeitig geht diese Verlängerung der Lebensjahre jedoch einher mit einer vermehrten Anzahl jener Jahre, die mit gesundheitlicher Beeinträchtigung erlebt werden.» Das ist richtig. Unsere Altersheime beherbergen eine grosse Zahl (sehr) pflegebedürftiger Personen. Dabei ist jedoch auch anzumerken, dass die Fortschritte in der Orthopädie oder in der Interventionskardiologie zum Beispiel vielen alten Menschen eine gute Lebensqualität verschaffen.

«Wir werden mit körperlichen und geistigen Begrenzungen konfrontiert, unsere Erwartungen gehen zurück. Ohne bewusste Wahl bemerken wir nicht, dass wir kaum noch Ambitionen haben.» Ich verstehe diese Argumentation bei jemandem, der vom amerikanischen Traum geprägt ist, andere (zu denen zähle ich mich auch) gehen jedoch davon aus, dass verringerte Energie und Effizienz, die mit dem Alterungsprozess einhergehen, gleichzeitig die Gelegenheit zur heiteren Reflexion und zu einer positiven Distanz bieten.

«Wichtig: Wie sollen sich andere an uns erinnern? Wir wollen nicht, dass wir als Last im Gedächtnis bleiben. Mit 75 erreichen wir jenen einzigartigen Punkt, an dem wir ein reiches Leben gelebt haben und - so bleibt zu hoffen - unseren Kindern und Enkeln Dinge mit auf den Weg gegeben haben, die sie in Erinnerung behalten. Später werden diese vitalen Erinnerungen verdrängt durch Verfallserscheinungen (Agonien) und unseren steigenden Bedarf an Pflege und Unterstützung.»

Wie also will Emanuel verfahren? «Ab 75 muss ich wirklich gute Gründe haben, um einen Arzt aufzusuchen. Ich werde keine kurativen, sondern nur noch palliative Massnahmen dulden. Ich will keine präventiven Kontrollen mehr. Also keine Koloskopien oder andere Tests. Ich bin nicht an einem PSA-Test interessiert. Keine Herzbelastungstests, keine Schrittmacher und kein Defibrillator» usw.
«Ich möchte» - so Emanuel in Le Temps [2] - «dass sich die Menschen Gedanken darüber machen, wie sie sich ihr Lebensende wünschen. Wenn wir wirklich darüber nachdenken, möchte nur eine kleine Gruppe wirklich bis dahin gelangen.» Darüber sind wir uns absolut einig. Wie aber sollen wir vorgehen? Es liegt klar auf der Hand, dass die von ihm vertretene Haltung den Pflegebedarf und die Abhängigkeiten vor dem Sterben nicht wesentlich verringert, also jene Phasen, die für die betroffene Person und die Angehörigen am schwersten zu ertragen sind. Oder geht er davon aus, dass die Erkenntnis unserer Ahnen von der «Pneumonie als der grossen Freundin der Greise» zu neuen «Ehren» gelangt, wenn er vor allem auf Antibiotika verzichtet? Bei uns lässt sich jedenfalls bereits eine Zurückhaltung gegenüber heroischer medizinischer Maximaleinsätze feststellen. Wahr ist aber auch - so Emanuel-, dass die «technologische Verpflichtung» (alles zu machen) in den USA immer noch ein grosses Hindernis bleibt.

Dabei fällt auf, dass sich dieser Autor seit Jahren gegen die Sterbehilfe bzw. die Euthanasie ausspricht. Ich selbst bin zwar nicht Mitglied bei Exit, der Thematik gegenüber jedoch aufgeschlossen. Möglicherweise erfolgt Sterbehilfe zwar nicht immer optimal, doch Berichten zufolge gestaltet sie sich nicht zwangsweise als herzzerreissendes, schweres Ereignis, sondern kann durchaus die Gelegenheit für Dialog oder Versöhnung bieten und in heiterer Gelassenheit erfolgen (u.a. auch, weil dem Ende ein Datum gesetzt ist). Und wie viele schätze ich die Vorstellung, dass ich im Falle von Abhängigkeit und irreversiblem Leid dabei unterstützt werde, meiner Existenz ein Ende zu bereiten. Der Ansatz von Emanuel hingegen lässt keine effizienten kurativen Massnahmen zu, und der Betroffene wartet darauf, dass sich sein Zustand bis zum Ende verschlechtert. Keine sehr tröstliche Aussicht.

Und als Letztes [1]: «Ich möchte nicht mehr als 75 Jahre alt werden. Meine Töchter und meine Freunde versuchen mich davon zu überzeugen, dass dies nicht richtig ist. Ich behalte mir daher das Recht vor, meine Meinung zu ändern, was alles in allem ja doch einfach nur besagen würde, dass ich über die 75 Jahre hinaus kreativ bleibe.» So ist man auch als bedeutende Persönlichkeit vor Wankelmut nicht gefeit.

1 Ezekiel JE. Why I Hope to Die at 75. Washington, D.C.: The Atlantic; Oktober 2014. (Artikel online verfügbar.)

2 «Pourquoi je souhaite mourir à 75 ans» (Interview von P. Minet). Le Temps. 9. Juni 2015; S. 14 